\title{
The Three-Nucleon System at Next-To-Next-To-Leading Order
}

\author{
L. Platter周 \\ Department of Physics and Astronomy, Ohio University, Athens, OH 45701, USA
}

(Dated: June 18, 2018)

\begin{abstract}
We calculate higher order corrections for the three-nucleon system up to next-to-next-to-leading within an effective field theory with contact interactions alone. We employ a subtraction formalism previously developed and for which it has been shown that no new three-body force counterterm is needed for complete renormalization up to this order. We give results for the neutron-deuteron phaseshifts and the triton binding energy. Our results are in very good agreement with experimental results and calculations using realistic nucleon-nucleon potentials.
\end{abstract}

PACS numbers: 11.80.Jy,21.10.Dr, 21.30.-x, 21.45.+v, 25.40.Dn, 27.10.+h

\section{INTRODUCTION}

The effective field theory (EFT) with contact interactions alone allows for a systematic calculation of lowenergy two-body observables in terms of the effective range parameters [1]. With the correct power counting it can be applied to systems with large scattering length $a$ and is an expansion in $R / a$, where $R$ denotes the range of the underlying interaction.

When applied to the three-body system with large scattering length, a three-body force [2] - or equivalently one subtraction - is needed to obtain cutoff independent results at leading order (LO). Although this fact seems to limit the predictive power of the EFT, it has been very successful in describing various atomic and nuclear lowenergy observables and has been used as a tool to understand universal properties of few-body systems with large scattering length [3, 4, 5].

To achieve high precision in three-body calculations higher order corrections have to be included. It is clear how to do this for the two-body system, however, in the three-body system it is not a priori obvious at which order an additional three-body counterterm has to be included. While Hammer and Mehen [ $\underline{6}$ ] showed that no additional three-body datum is needed for renormalization at next-to-leading order (NLO) and later works have come to the same conclusion, different conclusion have been reached for different renormalization methods at next-to-next-to-leading order (NNLO). While Bedaque et al. [7] and Barford and Birse [8] found that an additional energy-dependent three-body counterterm is needed for renormalization if an explicit three-body force is used and the cutoff $\Lambda$ is kept at $\sim 1 / R$, we showed recently [9] that within a subtraction formalism previously developed 10, 11], no additional three-body counterterm has to be introduced at NLO and NNLO for a consistent renormalization of observables if $\Lambda \gg 1 / R$. In this work, we computed also effective range corrections for the ${ }^{4} \mathrm{He}$ trimer system up to NNLO and achieved very good agreement with a previous calculation using a realistic atom-atom

*Electronic address: lplatter@phy.ohiou.edu potential. We showed that observables are cutoff independent for sufficiently large cutoffs and that effective range corrections to observables scale as expected. In this paper we will extend this work to the three-nucleon system and discuss the corresponding results. As the power counting in the neutron-deuteron quartet channel is well understood [12], we will focus here on the neutrondeuteron doublet channel.

In the following we will explain briefly the subtraction scheme which is used in this work to renormalize threebody observables, give results for the triton binding energy and scattering phaseshifts and discuss corrections up to NNLO to a universal correlation between the nucleondeuteron scattering length and the triton binding energy, known as the Phillips line. We will end this paper with a short conclusion and an outlook.

\section{THEORY}

At sufficiently low energies few-body systems interacting through short-range interactions can be described with an EFT built up from contact interactions alone. Employing an auxiliary field formalism [2] the neutrondeuteron system in the singlet channel can be described by the Lagrangian [5]

$$
\begin{gathered}
\mathcal{L}=N^{\dagger}\left(i \partial_{0}+\frac{\nabla^{2}}{2 M}\right) N-t_{i}^{\dagger}\left(\partial_{0}+\frac{\nabla^{2}}{4 M}-\Delta_{t}\right) t_{i} \\
-s_{j}^{\dagger}\left(\partial_{0}+\frac{\nabla^{2}}{4 M}-\Delta_{s}\right) s_{j} \\
-G_{3} N^{\dagger}\left(g_{t}^{2}\left(t_{i} \sigma_{i}\right)^{\dagger} t_{i^{\prime}} \sigma_{i^{\prime}}\right. \\
+\frac{1}{3} g_{t} g_{s}\left[\left(t_{i} \sigma_{i}\right)^{\dagger} s_{j} \tau_{j}+\text { h.c }\right] \\
\left.+g_{s}^{2}\left(s_{j} \tau_{j}\right)^{\dagger} s_{j^{\prime}} \tau_{j^{\prime}}\right)+\ldots,
\end{gathered}
$$

where $N$ represents the nucleon field and $t_{i}\left(s_{j}\right)$ are the dinucleon fields for the ${ }^{3} S_{1}\left({ }^{1} S_{0}\right)$ channels with the corresponding quantum numbers, respectively. The dots indicate more terms with more fields/derivatives.

The full two-body propagator $\tau$ is the result of dressing 
the bare dinucleon propagator by nucleon loops to all orders

$$
\tau_{\alpha}(E)=-\frac{2}{\pi m} \frac{1}{-\gamma_{\alpha}+\sqrt{-m E}+\frac{r_{\alpha}}{2}\left(\gamma_{\alpha}^{2}+m E\right)},
$$

where the index $\alpha=s, t$ indicates either the singlet or tiplet two-nucleon scattering channel. In this form the the two-body propagator has poles at energies outside the validity region of the EFT. Therefore the propagator cannot be used within the three-body integral equation for cutoffs $\Lambda>1 / r$ without employing additional techniques to subtract these unphysical poles. Instead of using the propagator in the form above we will expand it up to a given order in $R / a$

$$
\tau^{(n)}(E)=\frac{S^{(n)}(E)}{E+B_{d}}
$$

with $B_{d}=\gamma_{t}^{2} / m$ the deuteron binding energy and for $n<3 S^{(n)}$ is defined as

$$
S^{(n)}(E)=\frac{2}{\pi m^{2}} \sum_{i=0}^{n}\left(\frac{r}{2}\right)^{i}[\gamma+\sqrt{-m E}]^{i+1} .
$$

The set of integral equations for nucleon-deuteron scattering generated by this EFT (neglecting the three-body force for the beginning) is given by 5,11 .

$$
\begin{aligned}
K_{t t}^{(n)}\left(q, q^{\prime} ; E\right)=\mathcal{Z}_{t t} & \left(q, q^{\prime} ; E\right) \\
+ & \mathcal{P} \int_{0}^{\Lambda} \mathrm{d} q^{\prime \prime} q^{\prime \prime 2} \mathcal{Z}_{t t}\left(q, q^{\prime \prime} ; E\right) \\
& \times \tau_{t}^{(n)}\left(E-\frac{3}{4} \frac{q^{\prime \prime 2}}{m}\right) K_{t t}^{(n)}\left(q^{\prime \prime}, q^{\prime} ; E\right) \\
+ & \mathcal{P} \int_{0}^{\Lambda} \mathrm{d} q^{\prime \prime} q^{\prime \prime 2} \mathcal{Z}_{t s}\left(q, q^{\prime \prime} ; E\right) \\
& \times \tau_{s}^{(n)}\left(E-\frac{3}{4} \frac{q^{\prime \prime 2}}{m}\right) K_{s t}^{(n)}\left(q^{\prime \prime}, q^{\prime} ; E\right), \\
K_{s t}^{(n)}\left(q, q^{\prime} ; E\right)=\mathcal{Z}_{s t}( & \left.q, q^{\prime} ; E\right) \\
+ & \mathcal{P} \int_{0}^{\Lambda} \mathrm{d} q^{\prime \prime} q^{\prime \prime 2} \mathcal{Z}_{s t}\left(q, q^{\prime \prime} ; E\right) \\
& \times \tau_{t}^{(n)}\left(E-\frac{3}{4} \frac{q^{\prime \prime 2}}{m}\right) K_{t t}^{(n)}\left(q^{\prime \prime}, q^{\prime} ; E\right) \\
+ & \mathcal{P} \int_{0}^{\Lambda} \mathrm{d} q^{\prime \prime} q^{\prime \prime 2} \mathcal{Z}_{s s}\left(q, q^{\prime \prime} ; E\right) \\
& \times \tau_{s}^{(n)}\left(E-\frac{3}{4} \frac{q^{\prime \prime 2}}{m}\right) K_{s t}^{(n)}\left(q^{\prime \prime}, q^{\prime} ; E\right)
\end{aligned}
$$

where $m$ denotes the nucleon, $n$ the order of the calculation (for the following definitions it will always be assumed that $n<3$ ) and $\mathcal{Z}_{\alpha \beta}$ the Born amplitude

$$
\mathcal{Z}_{\alpha \beta}\left(q, q^{\prime} ; E\right)=-\lambda_{\alpha \beta} \frac{m}{q q^{\prime}} \log \left(\frac{q^{2}+q q^{\prime}+q^{\prime 2}-m E}{q^{2}-q q^{\prime}+q^{\prime 2}-m E}\right)
$$

with the iso-spin matrix $\lambda$ given by

$$
\lambda=\frac{1}{4}\left(\begin{array}{cc}
1 & -3 \\
-3 & 1
\end{array}\right)
$$

The set of integral equations in Eq.(5) is strongly cutoff dependent and a three-body force has to be introduced or equivalently a subtraction has to be performed to render observables cutoff independent. At threshold the integral equations are renormalized by noting that

$$
K_{t t}^{(n)}\left(0,0 ;-B_{d}\right)=\frac{3 m a_{3}}{8 \gamma \sum_{i=0}^{n}(\gamma r)^{n}}
$$

and subtracting this known quantity from Eq.(15)

$$
\begin{aligned}
& K_{t t}^{(n)}\left(q, 0 ;-B_{d}\right)-K_{t t}^{(n)}\left(0,0 ;-B_{d}\right) \\
& K_{s t}^{(n)}\left(q, 0 ;-B_{d}\right)-K_{t t}^{(n)}\left(0,0 ;-B_{d}\right) .
\end{aligned}
$$

After rewriting the resulting set of integral equations the half-off-shell threshold amplitude takes the following form [10, 11]

$$
\begin{aligned}
K_{t t}^{(n)}\left(q, 0 ;-B_{d}\right)= & K_{t t}^{(n)}\left(0,0 ;-B_{d}\right) \\
& +\left[\mathcal{Z}_{t t}\left(q, 0 ;-B_{d}\right)-\mathcal{Z}_{t t}\left(0,0 ;-B_{d}\right)\right] \\
& +\int_{0}^{\Lambda} \mathrm{d} q^{\prime \prime} q^{\prime \prime 2}\left[\mathcal{Z}_{t t}\left(q, q^{\prime \prime} ;-B_{d}\right)\right. \\
& \times \tau_{t}^{(n)}\left(-B_{d}-\frac{3 q^{\prime \prime 2}}{4 m}\right) K_{t t}^{(n)}\left(q^{\prime \prime}, 0 ;-B_{d}\right) \\
& +\int_{0}^{\Lambda} \mathrm{d} q^{\prime \prime} q^{\prime \prime 2}\left[\mathcal{Z}_{t s}\left(q, q^{\prime \prime} ;-B_{d}\right)\right. \\
& \times \tau_{s}^{(n)}\left(-B_{d}-\frac{3 q^{\prime \prime 2}}{4 m}\right) K_{s t}^{(n)}\left(q^{\prime \prime}, 0 ;-B_{d}\right), \\
K_{s t}^{(n)}\left(q, 0 ;-B_{d}\right)= & K_{t t}^{(n)}\left(0,0 ;-B_{d}\right) \\
& +\left[\mathcal{Z}_{s t}\left(q, 0 ;-B_{d}\right)-\mathcal{Z}_{t t}\left(0,0 ;-B_{d}\right)\right] \\
& +\int_{0}^{\Lambda} \mathrm{d} q^{\prime \prime} q^{\prime \prime 2}\left[\mathcal{Z}_{s t}\left(q, q^{\prime \prime} ;-B_{d}\right)\right. \\
& \times \tau_{s}^{(n)}\left(-B_{d}-\frac{3 q^{\prime \prime 2}}{4 m}\right) K_{s t}^{(n)}\left(q^{\prime \prime}, 0 ;-B_{d}\right) . \\
& \times \tau_{t}^{(n)}\left(-B_{d}-\frac{3 q^{\prime \prime 2}}{4 m}\right) K_{t t}^{(n)}\left(q^{\prime \prime}, 0 ;-B_{d}\right) \\
& +\int_{0}^{\Lambda} \mathrm{d} q^{\prime \prime} q^{\prime \prime 2}\left[\mathcal{Z}_{s s}\left(q, q^{\prime \prime} ;-B_{d}\right)\right. \\
& \\
& \\
& \\
& \\
&
\end{aligned}
$$

The amplitudes $K_{t t}^{(n)}\left(q, 0 ;-B_{d}\right)$ and $K_{s t}^{(n)}\left(q, 0 ;-B_{d}\right)$ are fully renormalized after the subtraction is performed. An essential point in obtaining the amplitudes at any energy and momentum is demanding that

$$
\begin{aligned}
& K_{t t}^{(n)}\left(q, 0 ;-B_{d}\right)=K_{t t}^{(n)}\left(0, q ;-B_{d}\right) \\
& K_{t s}^{(n)}\left(q, 0 ;-B_{d}\right)=K_{s t}^{(n)}\left(0, q ;-B_{d}\right) .
\end{aligned}
$$


Using resolvent identities, subtracted integral equations for any energy can be derived. For further details, the reader is advised to turn to 9, , 11].

The two-body parameters used throughout the rest of this work are given by

$$
\begin{aligned}
& \gamma_{t}^{-1}=4.317 \mathrm{fm}, \quad r_{t}=1.764 \mathrm{fm}, \\
& \gamma_{s}^{-1}=-25.04 \mathrm{fm}, \quad r_{s}=2.73 \mathrm{fm} .
\end{aligned}
$$

We will use the result of a recent neutron-deuteron scattering length measurement with $a_{3}=0.645 \pm 0.005 \mathrm{fm}$ as our three-body input 13.

\section{RESULTS}

The three-nucleon system has been previously considered within the EFT with contact interactions alone. Leading order results for phaseshift and the correlation between triton binding energy and neutron-deuteron scattering length were obtained in [5]. Bedaque et al. 7] calculated higher order corrections up to NNLO introducing an additional energy-dependent three-body force at this order. In this section we will demonstrate by explicit calculation that no additional three-body force is needed for renormalization and considerable improvement in results is achieved at NNLO. We have computed the triton binding energy at LO, NLO and NNLO using the neutron-deuteron scattering length as the threebody input parameter [13]. Our results are displayed in table [ When using the experimental value for the neutron-deuteron singlet scattering length $a_{3}=0.645 \mathrm{fm}$ as three-body input we obtain a triton binding energy of

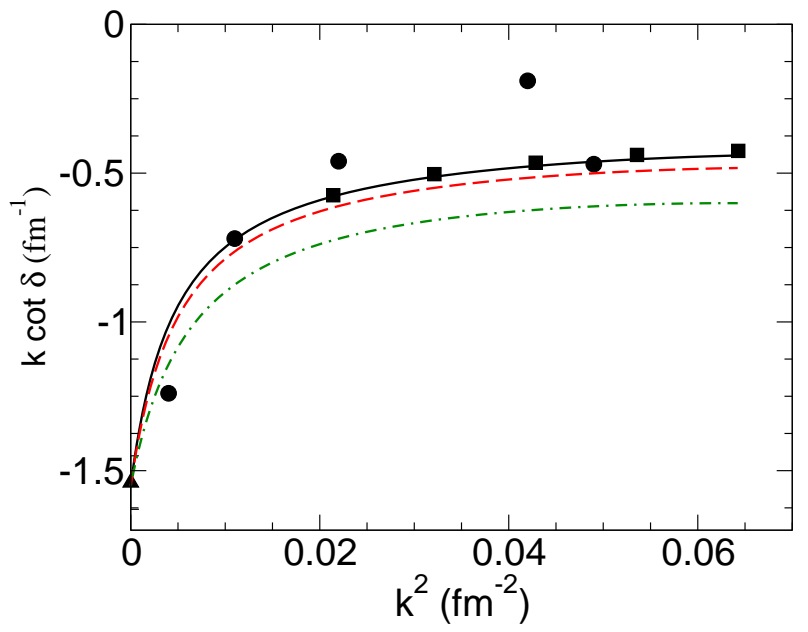

FIG. 1: (Color online) Phase shifts for neutron-deuteron scattering below the deuteron breakup at LO (dashed-dotted line), NLO (dashed line) and NNLO (solid line). The triangle is the result of the scattering length measurement of [13]. The circles are the results of the van Oers-Seagrave phaseshift analysis [14], and the squares denote a phaseshift calculation using a realistic nucleon-nucleon potential [15]

\begin{tabular}{|c|c||c|c||}
\hline & parameters & $a_{3}[\mathrm{fm}]$ & $B_{3}[\mathrm{Mev}]$ \\
\hline \hline $\mathrm{LO}$ & $a_{3}, \gamma_{t}, a_{s}$ & 0.645 & 8.08 \\
$\mathrm{NLO}$ & $a_{3}, \gamma_{t}, a_{s}, r_{t}, r_{t}$ & 0.645 & 8.19 \\
$\mathrm{NNLO}$ & $a_{3}, \gamma_{t}, a_{s}, r_{t}, r_{t}$ & 0.645 & 8.54 \\
\hline $\mathrm{EXP}$ & & 0.65 & 8.48 \\
\hline
\end{tabular}

TABLE I: EFT predictions for the triton binding energy up to NNLO using the neutron-deuteron scattering length as threebody input. The second column indicates which parameters were used at what order of the calculation. Energies and lengths are given in $\mathrm{MeV}$ and $\mathrm{fm}$, respectively.

$B_{3}=8.48 \mathrm{MeV}$ at NNLO which has to be compared to the experimental value $B_{3}^{\text {Exp }}=8.54 \mathrm{MeV}$. The results show a significant improvement with each order and are sufficiently close at NNLO to the experimental value to agree with a projected error at this order of approximately $\left(r_{t} \gamma_{t}\right)^{3} \sim 3 \%$. At these orders the error caused by the uncertainty in the effective range parameters is smaller than the error caused by $\mathrm{N}^{3} \mathrm{LO}$ corrections.

Using the set of integral equations in Eq.(15) with the expanded two-body propagator in Eq. (3) leads at LO, NLO and NNLO to the phaseshifts shown in Fig 1 For comparison we show in the same figure the results of a forty year old phaseshift analysis [14] and a theoretical calculation using a realistic nucleon-nucleon potential [15]. At higher order our results seem to describe the experimental data better but considering the age of the analysis and the fact that no errors are given for these data, the theoretical calculation by Kievsky et al. should be considered as the true benchmark test for our calculation. At NLO our results already lie significantly closer to this calculation and nearly perfect agreement is achieved at NNLO. It should be noted that our results at LO and NLO order agree with previous EFT calculations results given in [6, 7, 11]. We also achieve qualitative agreement at NNLO with Ref. [7], however, without employing an additional three-body counterterm.

A further way to illustrate the improvement in our results is to consider the Phillips line. The Phillips line is a universal feature of three-body systems with a large two-body scattering length and arises as a nearly linear correlation between the $1+2$ scattering length and the three-body binding energy. In Fig 2 we display our results for LO, NLO and NNLO and display also the experimental value. It is interesting to note that in contradistinction to the ${ }^{4} \mathrm{He}$ trimer system the Phillips line has not converged to a definite result yet [9]. The obvious reason for this is the rather large expansion coefficient which is roughly $\gamma_{t} r_{t} \sim 1 / 3$ while $\gamma r \sim 0.1$ in the ${ }^{4} \mathrm{He}$ system. The power counting derived in [9] is valid for cutoffs much larger than $1 / r$. Therefore, our results have been evaluated at corresponding cutoffs and in fact our results are fully converged with respect to $\Lambda$. The results given in this section are also numerically converged up to the digits displayed. Further, we also analyzed 


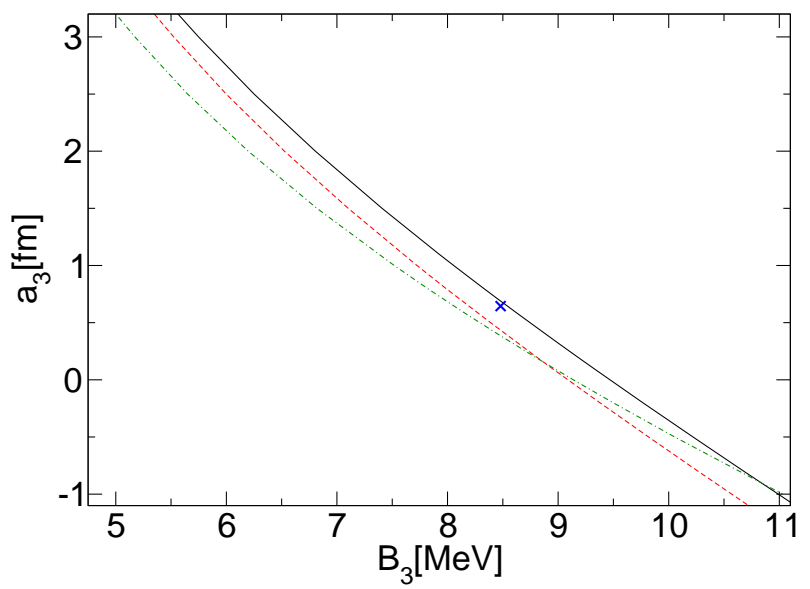

FIG. 2: (Color online) The Phillips line for leading (dotdashed line), next-to-leading (dashed line) and next-to-nextto-leading (solid line) order. The cross denotes the experimental value.

the convergence of three-body observables when using a different parametrisation for higher order two-body corrections called Z-matching [16, 17] and have found the same results up to the level of accuracy expected in an NNLO calculation.

\section{SUMMARY}

In this paper we have computed three-nucleon observables with the EFT with contact interactions alone up to NNLO using a subtraction scheme for renormalization. We have shown that the results for observables improve significantly at NNLO without performing an additional subtraction, $i, e$. without the introduction of a further energy dependent three-body counterterm. This improvement is in particular obvious if one considers the results for the binding energies and scattering phaseshifts simultaneously. Our value for the triton binding energy agrees very well with the experimental value and the results for the neutron-deuteron phaseshifts seem to be in nearly perfect agreement with a calculation using a realistic nucleon-nucleon potential. The results for the scattering phaseshifts agree qualitatively with previous calculations at NLO 6, 11] and NNLO 7], although a second three-body counterterm was included in [7]. In particular, these results seem to agree very well with an expected error of $(\gamma r)^{3} \sim 3 \%$ for an NNLO calculation. We have therefore presented further numerical evidence which supports the claim in Ref. [9] that calculations can be performed with exactly on three-body counterterm up to NNLO in the EFT with contact interactions alone.

Our analysis presented in [9] indicates that at $\mathrm{N}^{3} \mathrm{LO}$ an additional three-body input is needed for renormalization of observables. Therefore, NNLO can be also considered as the last order at which a prediction can be made for the Phillips line as it is a correlation gouverned by one three-body parameter.

Further possible applications of the subtraction formalism include the calculation of scattering observables above the breakup threshold and the coupling of external currents to the three-nucleon system, including the electromagnetic form factor beyond leading order [18].

\section{Acknowledgments}

I thank H.-W. Hammer and D. R. Phillips for stimulating discussions and comments on the manuscript. Furthermore, I thank Chubu University for its hospitality during partial completion of this work. This work was supported by the U.S. Department of Energy under grant DE-FG02-93ER40756.
[1] S. R. Beane, P. F. Bedaque, W. C. Haxton, D. R. Phillips, and M. J. Savage, in Encyclopedia of Analytic QCD, At the Frontier of Particle Physics, vol. 1, 133-269, edited by M. Shifman (World Scientific, Singapore, 2001).

[2] P. F. Bedaque, H.-W. Hammer, and U. van Kolck, Phys. Rev. Lett. 82, 463 (1999).

[3] E. Braaten and H. W. Hammer, Phys. Rept. 428, 259 (2006).

[4] L. Platter, H. W. Hammer and U. G. Meissner, Phys. Lett. B 607, 254 (2005).

[5] P. F. Bedaque, H. W. Hammer and U. van Kolck, Nucl. Phys. A 676, 357 (2000).

[6] H. W. Hammer and T. Mehen, Phys. Lett. B 516, 353 (2001).

[7] P. F. Bedaque, G. Rupak, H. W. Grießhammer and H. W. Hammer, Nucl. Phys. A 714, 589 (2003).

[8] T. Barford and M. C. Birse, J. Phys. A 38, 697 (2005).

[9] L. Platter and D. R. Phillips, arXiv:cond-mat/0604255
[10] H.-W. Hammer and T. Mehen, Nucl. Phys. A 690, 535 (2001).

[11] I.R. Afnan and D.R. Phillips, Phys. Rev. C 69, 034010 (2004).

[12] H. W. Griesshammer, Nucl. Phys. A 760, 110 (2005).

[13] T. C. Black et al., Phys. Rev. Lett. 90, 192502 (2003); K. Schön et al., Phys. Rev. C 67, 044005 (2003).

[14] W. T. H. van Oers and J. D. Seagrave,Phys. Lett. B 21, 526 (1967).

[15] A. Kievsky, S. Rosati, W. Tornow and M. Viviani, Nucl. Phys. A 607, 402 (1996).

[16] D. R. Phillips, G. Rupak and M. J. Savage, Phys. Lett. B 473, 209 (2000).

[17] H. W. Griesshammer, Nucl. Phys. A 744, 192 (2004).

[18] L. Platter and H. W. Hammer, Nucl. Phys. A 766, 132 (2006). 